\title{
BMJ Open Risk factors for 30-day in-hospital mortality for in-patient with stroke in sub-Saharan Africa: protocol for a systematic review and meta-analysis
}

\author{
Martin Ackah (D) , ${ }^{1}$ Cynthia Osei Yeboah, ${ }^{1}$ Louise Ameyaw ${ }^{2}$
}

To cite: Ackah M, Yeboah CO, Ameyaw L. Risk factors for 30-day in-hospital mortality for in-patient with stroke in sub-Saharan Africa: protocol for a systematic review and meta-analysis. BMJ Open 2021;11:e049927. doi:10.1136/ bmjopen-2021-049927

- Prepublication history and additional online supplemental material for this paper are available online. To view these files, please visit the journal online (http://dx.doi.org/10. 1136/bmjopen-2021-049927).

Received 08 February 2021 Accepted 09 July 2021

Check for updates

(C) Author(s) (or their employer(s)) 2021. Re-use permitted under CC BY-NC. No commercial re-use. See rights and permissions. Published by BMJ.

${ }^{1}$ Department of Physiotherapy, Korle Bu Teaching Hospital, Accra, Greater Accra, Ghana

${ }^{2}$ School of public Health,

University of Ghana College of Health Sciences, Accra, Greater Accra, Ghana

Correspondence to

Martin Ackah;

martinackah10@gmail.com

\section{ABSTRACT}

Introduction While individual studies have reported on in-hospital stroke mortality rates in sub-Saharan Africa (SSA), the estimates are highly variable and inconclusive, buttressing the need for precise and reliable estimations. To overcome these inconsistencies, a well-structured systematic review and meta-analytical models are necessary. However, to the best of our knowledge, there is no published systematic review and meta-analysis on risk factors for 30-day mortality for in-hospital patients with stroke in SSA.

Method and analysis We will include all retrospective and prospective facility-based observational studies reporting on the incidence and/or risk factors for inhospital stroke mortality in SSA. Electronic databases such as PubMed, Google scholar and Africa Journal Online (AJOL) will be searched for potentially relevant studies on in-hospital stroke mortality and risk factors in SSA. The search will be limited to studies conducted from January 1990 to December 2020. Two independent authors will screen titles and abstract to find studies that meet the prespecified eligibility criteria for inclusion in the review. The incidence of 30-day in hospital stroke mortality will be pooled. Meta-regression will be used to assess the factors associated with in-hospital stroke mortality in SSA. If possible, subgroup analysis will be performed based on subregion, publication year and study design, and quality score to determine possible source of heterogeneity. If possible, a sensitivity analysis will be performed to determine the robustness of the estimates obtained from the meta-analysis.

Ethics and dissemination Ethical approval is not required as this is a secondary research and will use reported data in scientific literature. A full manuscript will be submitted to a reputable peer-review journal for publication.

PROSPERO registration number CRD42021227367.

\section{INTRODUCTION}

Stroke is a major cause of death and injury, and poststroke treatment costs are a significant economic burden worldwide. ${ }^{2}$ High-income countries have seen rapid and significant reduction in stroke incidence, and long-term survival as a result of expanded use of preventive therapies
Strengths and limitations of this study

To the best of the authors' knowledge, this is the first systematic review and meta-analysis on risk factors for 30-day in-hospital stroke mortality rate in subSaharan Africa.

- Sensitivity analyses will be performed to determine the robustness of the estimates obtained from the meta-analysis.

- We would incorporate well-validated systematic review and meta-analysis technique that are completely consistent with existing international standards and recommendations.

- Due to regional and geographical differences, there can be variations across studies, therefore, we plan to conduct robust sub-group analyses to detect any subgroup effects.

- Papers/articles that have only been published in English would be considered, which could introduce publication bias.

and significant decreases in premorbid risk factors. ${ }^{3-5}$ Nonetheless, most sub-Saharan Africa countries (SSA) are unable to say same. ${ }^{6}$

The incidence of stroke is rising in lowincome and middle-income countries (LMICs) in SSA countries, and research has shown that between 2002 and 2020, stroke mortality in SSA was tripled. ${ }^{78}$ For instance, community-based SSA studies indicate that $5 \%-10 \%$ of all deaths are caused by stroke, partially due to poor health system and rising rates of hypertension. ${ }^{9}{ }^{10}$ In addition, LMICs account for $85 \%$ of all stroke deaths, as well as $87 \%$ of total losses due to stroke measured in disability-adjusted life-years which total 72 million per year worldwide ${ }^{11}$

In SSA, epidemiological studies have shown that in-hospital stroke mortality rates varied from $18 \%$ in Ethiopia to $43 \%$ in Ghana. ${ }^{8} 12$ SSA countries have insufficient resources for acute medical and rehabilitation care for stroke, therefore, comprehensive and 
pragmatic preventive efforts directed at risk factors are of utmost importance to curtail the burden. ${ }^{13}$ In the same vein, early intervention on in-patient with stroke identified with a high risk of mortality may increase the survival rate. ${ }^{14}$ It is, therefore, imperative to identify risk factors for 30-day in-hospital mortality for in-patients with stroke in SSA. The proportion of patients who die within 30 days from the time of admission to the time of death among all patients hospitalised with stroke is referred to as 30-day in-hospital stroke mortality.

While individual studies have reported on in-hospital stroke mortality rates in SSA, the estimates are highly variable and inconclusive, buttressing the need for precise and reliable estimations. To overcome these inconsistencies, a well-structured systematic review and meta-analytical models are necessary. However, to the best of our knowledge, there is no published systematic review and meta-analysis on risk factors for 30-day mortality for in-hospital patients with stroke in SSA.

With this in mind, the study seeks to systematically review empirical evidence on risk factors for 30-day mortality for in-hospital patients with stroke in SSA. It is important for healthcare providers to learn about the risk factors associated with in-hospital stroke 30-day mortality in order to prepare for future patient care as well as to optimise hospital staffing and necessary skills in SSA.

\section{Review questions}

- What is the incidence for 30-day mortality rates for in-patients with stroke in SSA?

- What are the risk factors for 30-day mortality rates for in-patients with stroke in SSA?

\section{Objectives}

- Primary objective: To determine the incidence for 30-day mortality rates for in-patients with stroke in SSA.

- Secondary objective: To assess the risk factors for 30-day mortality for in-patients with stroke in SSA.

\section{METHODS}

\section{Patient and public involvement}

Patients and/or the public were not involved in the design, or conduct, or reporting, or dissemination plans of this research.

\section{Ethics and dissemination}

Ethical approval is not required as this is a secondary research and will use reported data in scientific literature. A full manuscript will be submitted to a reputable peer-review journal for publication.

\section{Protocol registration and best practices}

This systematic review and meta-analysis will follow strict adherence to the guidelines of the Preferred Reporting Items for Systematic Reviews and Meta-Analyses Protocols (PRISMA-P) ${ }^{15}$ (online supplemental file 2).

\section{Eligibility}

\section{Types of studies}

All retrospective and prospective facility-based observational studies reporting on incidence and/or risk factors for in-hospital stroke mortality and case fatality in SSA countries. Also, if any of the countries in SSA have a public reporting of in-hospital 30-day mortality from eventual published quality indicators, such outcome will be included to help contribute to the identification and understanding of risk factors. Animal studies, reviews, commentaries, conference papers and letter to the editor will be excluded.

\section{Types of participants}

Studies from SSA countries involving in-hospital patients with stroke. The review will consider all age groups.

\section{Types of outcome measures}

The primary outcome is the in-hospital stroke 30-day mortality in SSA and secondary outcome is the risk factors for in-hospital mortality in SSA. However, if any study reports on out-of-hospital mortality, it will be extracted and reported separately.

\section{Data source and search strategies}

Primary electronic search in English on the incidence and risk factors for in-hospital stroke case-fatality rate in SSA will be conducted in MEDLINE via PubMed, Google Scholar and AJOL. The search will be limited to studies conducted from January 1990 to December 2020. Table 1 displays the main search term and approaches (online supplemental file 1). The abstracts of all eligible papers will then be reviewed and full articles will be accessed through PubMed, Google Scholar, and AJOL. Reference lists of papers that fulfil the eligibility requirements of the study will be reviewed to identify additional studies not included in our electronic search. To ensure that potential studies that will be missed by electronic searching are included, experts will be consulted.

\section{Screening and selecting studies}

Two authors will screen titles and abstract independently to find studies that meet the pre-specified eligibility criteria for inclusion in the review. Full texts of all potentially relevant studies will be accessed and assessed in detail in a similar manner. A third reviewer will be available to resolve any discrepancies between the two independent assessors. A screening guide will be used to ensure that independent reviewers apply the selection criteria reliably. Authors whose full-text documents are not available via a variety of internet-based sources will be contacted directly through the corresponding authors to provide them to help make the final decision about inclusion. If vital information needed to make the inclusion decision is not obtained, the article will be excluded. Mendeley reference manager will be used to deduplicate studies. 


\begin{tabular}{|c|c|}
\hline Search \# & Search term \\
\hline 1) & In-hospital OR in-patient \\
\hline 2) & $\begin{array}{l}\text { Stroke OR cerebrovascular accident OR CVA OR } \\
\text { cerebral infarction OR Ischemic stroke OR Lacuna } \\
\text { stroke OR cerebral hemorrhage OR haemorrhagic } \\
\text { stroke }\end{array}$ \\
\hline 3) & 1 AND 2 \\
\hline 4) & $\begin{array}{l}\text { Mortality OR 30-day mortality OR death OR case- } \\
\text { fatality }\end{array}$ \\
\hline 5) & 3 AND 4 \\
\hline 6) & risk factors OR associated factors \\
\hline 7) & 5 OR 6 \\
\hline 8) & sub-Saharan Africa \\
\hline 9) & $\begin{array}{l}\text { Angola OR Benin OR Botswana OR Burkina Faso } \\
\text { OR Burundi OR Cameroon OR Cape Verde OR } \\
\text { Central African Republic OR Chad OR Comoros } \\
\text { OR Congo OR Cote d'Ivoire OR Djibouti OR } \\
\text { Equatorial Guinea OR Ethiopia OR Gabon OR } \\
\text { The Gambia OR Ghana OR Guinea OR Guinea- } \\
\text { Bissau OR Kenya OR Lesotho OR Liberia OR } \\
\text { Madagascar OR Malawi OR Mali OR Mauritania } \\
\text { OR Mauritius OR Mozambique OR Namibia OR } \\
\text { Niger OR Nigeria OR Rwanda OR Sao Tome and } \\
\text { Principe OR Senegal OR Seychelles OR Sierra } \\
\text { Leone OR Somalia OR South Africa OR Sudan } \\
\text { OR Swaziland OR Tanzania OR Togo OR Uganda } \\
\text { OR Zaire OR Zambia OR Zimbabwe }\end{array}$ \\
\hline 10) & 8 OR 9 \\
\hline 11) & Limit to January, 1990-December,2020 \\
\hline 12) & Limit to Humans \\
\hline 13) & 10 AND 11 AND 12 \\
\hline
\end{tabular}

\section{Data extraction and management}

Two independent assessors will extract the data from the eligible published articles using a pretested and standardised excel spreadsheet. Data such as the last name of the first author's name, year of publication, study country, study design, sample size, mortality rate, risk factors for in-hospital stroke case-fatality, severity measure, type of stroke as well as the demographic information (ie, sex, age, etc) will be extracted. Missing data will be addressed by contacting the corresponding author for insufficient or unclear data. If possible, corresponding authors will be asked to provide us with the raw data to extract the missing data.

\section{Outcome and operationalisation}

A 30-day in-hospital stroke mortality is operationally defined as the proportion or standardised hospital mortality based on the number of patients who die within 30 days from the time of admission to the time of death among all patients hospitalised with stroke. In this study, a risk factor is defined as a set of variables that are linked to or cause 30-day death in hospitalised stroke patients in SSA. For example, patient-related factors that may increase mortality in stroke include poor control of major risk factors to stroke such as hypertension, obesity, smoking, heart disease and diabetes. Hospital-related factors such as availability of a stroke unit, availability of an intensive care unit and the capacities of the emergency unit. Treatment delays (ie, waiting times, time to get to hospital from onset of symptoms). The severity of stroke and length of stay may also influence 30-day mortality, etc.

\section{Risk of bias and quality assessment}

The Newcastle-Ottawa Quality Assessment tool adapted for cross-sectional studies will be used to assess the quality of the retrieved studies ${ }^{16}$. The purpose of the assessment will be to determine the internal and external validity of the studies and to minimise risk of bias.

\section{Data synthesis}

Extracted data will be exported into Stata (V.16; Stata) from Microsoft excel 2013 for all analyses. The PRISMA flow chart (figure 1) will be used to summarised the selection process. When considerable homogeneity exists among the studies, the incidence of 30-day in-hospital stroke mortality in SSA will be pooled. This will be visually represented using the forest plot. The presence of heterogeneity among studies will be quantified by estimating variance using both Cochrane's $Q$ statistics and the $I^{2}$ statistics. ${ }^{17}$ The $\mathrm{I}^{2}$ takes values between $0 \%$ and $100 \%$, and a value of $0 \%$ indicates absence of heterogeneity. $\mathrm{I}^{2}$ will be interpreted based on Higgins and Thompson classification, percentages of $25 \%, 50 \%$ and $75 \%$ will be considered as low, moderate and high heterogeneity, respectively. ${ }^{17}$

Meta-regression will be used to assess the factors associated with in-hospital stroke 30-day mortality in SSA. If possible, subgroup analysis will be performed based on subregion (West Africa vs East Africa vs Southern Africa), publication year and study design (prospective vs retrospective), and quality score (low risk vs moderate risk vs high risk of bias) to determine possible source of heterogeneity.

If possible, a sensitivity analysis will be performed to determine the robustness of the estimates obtained from the meta-analysis. We will do sensitivity analysis on the quality of the studies included in the systematic review and meta-analysis, that is, studies with low quality score will initially be excluded to check their direction and impact on the overall (pooled) estimate and finally leave one out sensitivity analysis will be performed. Publication bias will be checked by the funnel plot and Egger's test. Furthermore, trim and fill analysis will be used to adjust for publication bias using Duval and Tweedie's method ${ }^{18}$ in case publication bias exist.

In event where meta-analysis is not possible due to considerable heterogeneity and low-quality studies, narrative systematic review will be presented. 


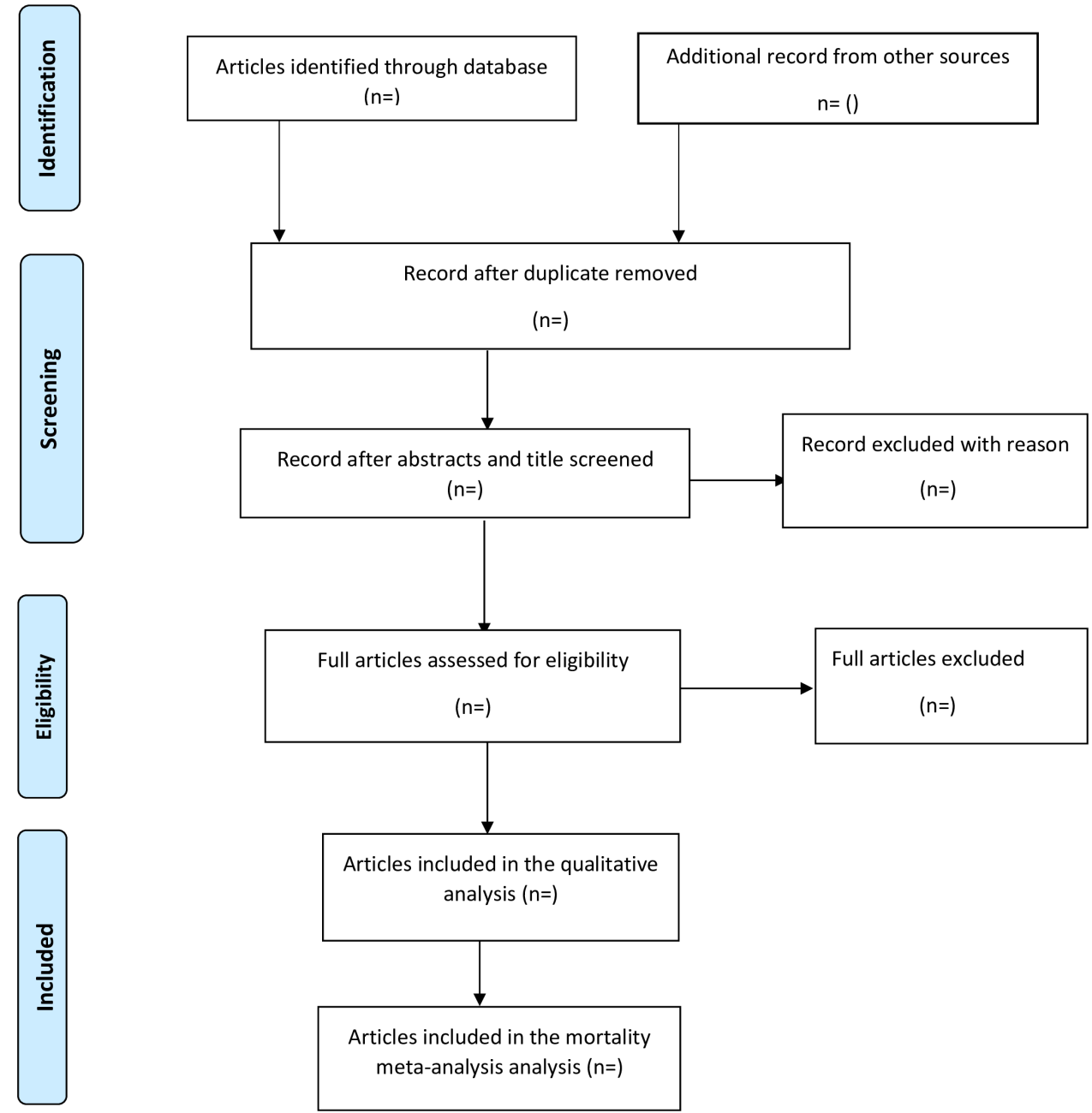

Figure 1 Preferred Reporting Items for Systematic Reviews and Meta-Analyses Protocols flow chart for study selection.

\section{Grading the quality of evidence}

The quality of evidence for all studies will be assessed using the Grading of Recommendations Assessment, Development and Evaluation (GRADE) working group methodology. The following domains will be assessed: risk of bias, consistency, directness, precision, publication bias and additional points. The assessments will be classified into four levels: high, moderate, low or very low. ${ }^{19}$ Two independent reviewers will assess the GRADE and disagreement will be resolved through discussion.

\section{Expected key results and discussion}

Globally, stroke is the third leading cause of death. ${ }^{20}$ The bulk of these deaths from strokes are found in LMICs. In these nations, deaths account for up to $87 \%$ of all stroke fatalities. ${ }^{21}$ This elevated death toll is much greater in SSA. ${ }^{21}$ To the best of the authors' knowledge, there is no comprehensive systematic review and meta-analysis on in-hospital stroke case fatality exist in SSA. Hence, the primary aim of this review is to determine the incidence of in-hospital stroke 30-day mortality in SSA. Secondary objective is to assess the risk factors for in-hospital stroke mortality in SSA.
Acknowledgements Sincere thanks to Nana Abena Nyamedo Yeboah for critical reading of the manuscript and comments.

Contributors MA conceived the study, drafted the manuscript, critically revised the manuscript for methodological and intellectual content. COY drafted the manuscript, critically revised the manuscript for methodological and intellectual content. LA critically revised the manuscript for methodological and intellectual content. All authors approved the final manuscript. MA is the guarantor of the review.

Funding The authors have not declared a specific grant for this research from any funding agency in the public, commercial or not-for-profit sectors.

Competing interests None declared.

Patient consent for publication Not required.

Provenance and peer review Not commissioned; externally peer reviewed.

Supplemental material This content has been supplied by the author(s). It has not been vetted by BMJ Publishing Group Limited (BMJ) and may not have been peer-reviewed. Any opinions or recommendations discussed are solely those of the author(s) and are not endorsed by BMJ. BMJ disclaims all liability and responsibility arising from any reliance placed on the content. Where the content includes any translated material, BMJ does not warrant the accuracy and reliability of the translations (including but not limited to local regulations, clinical guidelines, terminology, drug names and drug dosages), and is not responsible for any error and/or omissions arising from translation and adaptation or otherwise.

Open access This is an open access article distributed in accordance with the Creative Commons Attribution Non Commercial (CC BY-NC 4.0) license, which permits others to distribute, remix, adapt, build upon this work non-commercially, and license their derivative works on different terms, provided the original work is 
properly cited, appropriate credit is given, any changes made indicated, and the use is non-commercial. See: http://creativecommons.org/licenses/by-nc/4.0/.

ORCID iD

Martin Ackah http://orcid.org/0000-0002-5045-1104

\section{REFERENCES}

1 GBD 2016 Stroke Collaborators. Global, regional, and national burden of stroke, 1990-2016: a systematic analysis for the global burden of disease study 2016. Lancet Neurol 2019;18:439-58.

2 Rajsic S, Gothe H, Borba HH, et al. Economic burden of stroke: a systematic review on post-stroke care. Eur J Health Econ 2019;20:107-34

3 Rothwell PM, Coull AJ, Giles MF, et al. Change in stroke incidence, mortality, case-fatality, severity, and risk factors in Oxfordshire, UK from 1981 to 2004 (Oxford vascular study). Lancet 2004;363:1925-33.

4 Peltonen M, Stegmayr B, Asplund K. Time trends in long-term survival after stroke. Stroke 1998;29:1358-65.

5 Eriksson M, Carlberg B, Eliasson M. The disparity in long-term survival after a first stroke in patients with and without diabetes persists: the Northern Sweden MONICA study. Cerebrovasc Dis 2012;34:153-60.

6 Sanya E, Wahab KW, Bello $\mathrm{AH}$, et al. In-hospital stroke mortality and its predictors within one month of ictus: result from a tertiary hospital in Ilorin, middle belt Nigeria. Sub-Saharan Afr J Med 2015;2:165-9.

7 World Health Organization. Towards a who long-term strategy for prevention and control of leading chronic diseases, 2004.

8 Kaduka L, Muniu E, Oduor C, et al. Stroke mortality in Kenya's public tertiary hospitals: a prospective Facility-Based study. Cerebrovasc Dis Extra 2018;8:70-9.

9 Agyemang C, Attah-Adjepong G, Owusu-Dabo E, et al. Stroke in Ashanti region of Ghana. Ghana Med J 2012;46:12-17.
10 Sanuade OA, Dodoo FN-A, Koram K, et al. Prevalence and correlates of stroke among older adults in Ghana: evidence from the study on global ageing and adult health (SAGE). PLoS One 2019;14:e0212623-17.

11 Dabilgou AA, Dravé A, Kyelem JMA, Marie J, et al. Frequency and mortality risk factors of acute ischemic stroke in emergency department in Burkina Faso. Stroke Res Treat 2020;2020:9745206.

12 Alene M, Assemie MA, Yismaw L, et al. Magnitude of risk factors and in-hospital mortality of stroke in Ethiopia: a systematic review and meta-analysis. BMC Neurol 2020;20:1-10.

13 Owolabi MO, Sarfo F, Akinyemi R, et al. Dominant modifiable risk factors for stroke in Ghana and Nigeria (siren): a case-control study. Lancet Glob Health 2018;6:e436-46.

14 Zhang R, Wang Y, Fang J, et al. Worldwide 1-month case fatality of ischaemic stroke and the temporal trend. Stroke Vasc Neurol 2020;5:353-60.

15 Shamseer L, Moher D, Clarke M, et al. Preferred reporting items for systematic review and meta-analysis protocols (PRISMA-P) 2015 elaboration and explanation. BMJ 2015;349:g7647-25.

16 Luchini C, Stubbs B, Solmi M, et al. Assessing the quality of studies in meta-analyses: advantages and limitations of the Newcastle Ottawa scale. World J Metaanal 2017;5:80.

17 Higgins JPT, Thompson SG. Quantifying heterogeneity in a metaanalysis. Stat Med 2002;21:1539-58.

18 Duval S, Tweedie R. Trim and fill: a simple funnel-plot-based method of testing and adjusting for publication bias in meta-analysis. Biometrics 2000;56:455-63.

19 Balshem H, Helfand M, Schünemann HJ, et al. Grade guidelines: 3. rating the quality of evidence. J Clin Epidemiol 2011;64:401-6.

20 Feigin VL, Lawes CMM, Bennett DA, et al. Worldwide stroke incidence and early case fatality reported in 56 population-based studies: a systematic review. Lancet Neurol 2009;8:355-69.

21 Ekeh B, Ogunniyi A, Isamade E, et al. Stroke mortality and its predictors in a Nigerian teaching hospital. Afr Health $\mathrm{Scl}$ 2015;15:74-18. 